\title{
SUPPLEMENT TO: TENSOR DECOMPOSITIONS AND SPARSE LOG-LINEAR MODELS
}

\author{
By James E. Johndrow*, Anirban Bhattacharya and David B. \\ DUNSON* \\ Duke University ${ }^{\dagger}$ and Texas A\&M University
}

\section{Supplemental Results.}

\subsection{Proof of Remark 3.4. Let}

$\mathscr{H}_{g}=\left\{H \in \mathscr{H}:\right.$ there exists no $H^{*} \in \mathscr{H}$ for which $H_{j}^{*} \subset H_{j}$ for every $\left.j \in U\right\}$.

We claim that if $H \in \mathscr{H}_{g}$, the only partitions satisfying conditional independence with fewer elements than $\mathcal{P}_{H}^{0}$ are those in which events in one or more collections $\mathcal{P}_{H, l}^{\alpha}$ are replaced with sets $\mathcal{B}^{\alpha}$ as in the proof of (14). We demonstrate that replacement of any other collections of events by their union gives an event conditional upon which $y_{[1: p]}$ are not independent.

Choose $H \in \mathscr{H}_{g}$. Let $B_{1}, B_{2} \in \mathcal{P}_{H}^{0}$ for which there exists no $l \in V, \boldsymbol{\alpha} \in \mathcal{A}$ such that $B_{1}$ and $B_{2}$ belong to the same collection $\mathcal{P}_{H, l}^{\alpha}$. Set $A=B_{1} \cup B_{2}$, $J_{1}=\left\{j: H_{j}^{c} \cap A=A\right\}, J_{2}=\left\{j: C_{j} \cap A=A,\left|C_{j}\right|>1, C_{j} \neq H_{j}^{c}\right\}$, $J=J_{1} \cup J_{2}, J^{(-l)}=J \backslash\{l\}$, and $\bar{J}=V \backslash J$. Then by construction

$$
A=\underset{k \in \bar{J}}{\chi}\left\{c_{k}\right\} \times \underset{j \in J_{1}}{\chi} \bar{H}_{j} \times \underset{j \in J_{2}}{X} C_{j}
$$

The conditions imply that $J_{2}$ must be nonempty. Otherwise, there would exist $c_{l} \in H_{l}$ for some $l \in V$ such that if we set $H_{l}=H_{l} \backslash\left\{c_{l}\right\}$ and $H^{*}=\left\{\left\{H_{j}\right.\right.$ : $\left.j \neq l\}, H_{l}\right\}$ then $T_{C_{\theta}, H^{*}}=C_{\theta}$ and $\sum_{j}\left|H_{j}^{*}\right|<\sum_{j}\left|H_{j}\right|$, contradicting $H \in$ $\mathscr{H}_{g}$. Without loss of generality, we $\operatorname{suppose}_{\max _{j}}\{j \in \bar{J}\}<\min _{j}\left\{j \in J_{1}\right\}=q$ and $\max _{j}\left\{j \in J_{1}\right\}<\min _{j}\left\{j \in J_{2}\right\}=r$. Define

$$
\overline{\boldsymbol{C}}_{E}=\underset{j \in\left(E \cap J_{1}\right)}{\chi} H_{j}^{c} \times \underset{j \in\left(E \cap J_{2}\right)}{X} C_{j} .
$$

*This research was partially support by grants ES017436, ES020619, and ES017240 from the National Institute of Environmental Health Sciences (NIEHS) of the US National Institutes of Health. The second author acknowledges support for this project from the Office of Naval Research (ONR BAA 14-0001). 
From the proof of (13) in Theorem 3.2, we need to show that with this choice of $A$, there exists a $i \in \tilde{\mathcal{I}}_{V}$ such that

$$
\sum_{\boldsymbol{\alpha}^{(q)} \in \overline{\boldsymbol{C}}_{J^{(-q)}}} \cdots \sum_{\boldsymbol{\alpha}^{(p)} \in \overline{\boldsymbol{C}}_{J^{(-p)}}} \prod_{l \in J} \frac{\pi_{c_{k} i_{l} \alpha_{j}^{(l)}}^{\left(\bar{J}\{l l\}, J^{(-l)}\right)}}{\pi_{\boldsymbol{i}}} \neq \sum_{\boldsymbol{\alpha}_{q} \in \overline{\boldsymbol{C}}_{J}} \cdots \sum_{\boldsymbol{\alpha}_{p-1} \in \overline{\boldsymbol{C}}_{J}} \prod_{l \in J^{(-p)}} \frac{\pi_{c_{k} \alpha_{l j}}^{(\bar{J}, J)}}{\pi_{\boldsymbol{i}}}
$$

Introducing additional notation, let

$$
\begin{aligned}
\mathcal{E}_{1} & =\{E: E \subset(\bar{J} \cup\{j\}), j \in J, E \cap J \neq \varnothing\}, \\
\mathcal{E}_{2} & =\left\{E: E \cap J_{2} \neq \varnothing,|E \cap J| \geqslant 2\right\}, \\
\mathcal{E}_{1}^{(-l)} & =\left\{E: E \subset(\bar{J} \cup\{j\}), j \in J^{(-l)}, E \cap J \neq \varnothing\right\}, \text { and } \\
\mathcal{E}_{2}^{(-l)} & =\left\{E: l \notin E, E \cap J_{2} \neq \varnothing,|E \cap J| \geqslant 2\right\} .
\end{aligned}
$$

Let $\boldsymbol{i}^{(l)}$ be defined as in the proof of (13), and make the additional definition that $\tilde{\boldsymbol{i}}^{(l)}$ is the cell such that $\tilde{i}_{k}^{(l)}=i_{k}^{(l)}$ if $k \neq l$ and $\tilde{i}_{k}^{(l)}=c_{k}$ if $k=l$. Clearly

$$
\begin{aligned}
\frac{\pi_{c_{k} i_{l} \alpha_{j}^{(l)}}^{\left(\bar{J},\{l\}, J^{(-l)}\right)}}{\pi_{\boldsymbol{i}}} & =\exp \left[\sum_{E \in\left(\mathcal{E}_{1}^{(-l)} \cup \mathcal{E}_{2}\right)}\left\{\theta_{E}\left(\tilde{\boldsymbol{i}}_{E}^{(l)}\right)-\theta_{E}\left(\boldsymbol{i}_{E}\right)\right\}\right] \\
& =\exp \left[\sum_{E \in \mathcal{E}_{1}^{(-l)}}\left\{\theta_{E}\left(\boldsymbol{i}_{E}^{(l)}\right)-\theta_{E}\left(\boldsymbol{i}_{E}\right)\right\}\right] \exp \left[\sum_{E \in \mathcal{E}_{2}}\left\{\theta_{E}\left(\tilde{\boldsymbol{i}}_{E}^{(l)}\right)-\theta_{E}\left(\boldsymbol{i}_{E}\right)\right\}\right],
\end{aligned}
$$

since the $l$ coordinate of the cell $\tilde{\boldsymbol{i}}^{(l)}$ is irrelevant when summing over $E \in$ $\mathcal{E}^{(-l)}$, and

$$
\begin{aligned}
& \frac{\pi_{c_{k} \alpha_{l j}}^{(\bar{J}, J)}}{\pi_{\boldsymbol{i}}}=\exp \left[\sum_{E \in\left(\mathcal{E}_{1} \cup \mathcal{E}_{2}\right)}\left\{\theta_{E}\left(\boldsymbol{i}_{E}^{(l)}\right)-\theta_{E}\left(\boldsymbol{i}_{E}\right)\right\}\right] \\
& (\mathrm{S} .2) \quad=\exp \left[\sum_{E \in \mathcal{E}_{1}}\left\{\theta_{E}\left(\boldsymbol{i}_{E}^{(l)}\right)-\theta_{E}\left(\boldsymbol{i}_{E}\right)\right\}\right] \exp \left[\sum_{E \in \mathcal{E}_{2}}\left\{\theta_{E}\left(\boldsymbol{i}_{E}^{(l)}\right)-\theta_{E}\left(\boldsymbol{i}_{E}\right)\right\}\right] .
\end{aligned}
$$

For any $E \in \mathcal{E}_{1}$, we can proceed exactly as in the proof of (13), and therefore we consider the second terms in (S.1) and (S.2). Since $p \in J_{2}$, there must exist $E^{*} \subset J$ with $p \in E^{*}$ such that for some $\boldsymbol{i}^{*} \in \tilde{\mathcal{I}}_{V}, \theta_{E}\left(\boldsymbol{i}_{E}^{*}\right) \neq 0$. Without loss of generality, suppose $E^{*}=\{p, q\}$ where as above $q=p-|J|$. Let $\tilde{I}_{V}^{(1)}$ be 
the set of cells corresponding to the event $B_{1}$ and $\tilde{I}_{V}^{(2)}$ those corresponding to $B_{2}$. One of these sets must have $i_{q}=i_{q}^{*}$ and $i_{p}=i_{p}^{*}$ for every element, since $\theta_{E}\left(\boldsymbol{i}_{E}^{*}\right) \neq 0$. Take this to be $\tilde{\mathcal{I}}_{V}^{(1)}$, in which case no $\boldsymbol{i} \in \tilde{\mathcal{I}}_{V}^{(2)}$ has both $i_{q}=i_{q}^{*}$ and $i_{p}=i_{p}^{*}$. Otherwise, there exists $l \in V$ such that $B_{1}$ and $B_{2}$ belong to the same collection $\mathcal{P}_{H, l}^{\alpha}$, which is false by choice of $B_{1}$ and $B_{2}$.

Without loss of generality, suppose that $i \in \tilde{\mathcal{I}}_{V}^{(2)}$ implies $i_{q} \neq i_{q}^{*}$ and take $c_{q} \neq i_{q}^{*}$. Then $\theta_{E^{*}}\left(\tilde{\boldsymbol{i}}_{E^{*}}^{(l)}\right) \neq \theta_{E^{*}}\left(\boldsymbol{i}_{E^{*}}^{(l)}\right)$ whenever $\alpha_{l q}=i_{q}^{*}$, in which case we have

$$
\prod_{l \in J} \exp \left[\sum_{E \in \mathcal{E}_{2}}\left\{\theta_{E}\left(\tilde{\boldsymbol{i}}_{E}^{(l)}\right)-\theta_{E}\left(\boldsymbol{i}_{E}\right)\right\}\right] \neq \prod_{l \in J(-p)} \exp \left[\sum_{E \in \mathcal{E}_{2}}\left\{\theta_{E}\left(\boldsymbol{i}_{E}^{(l)}\right)-\theta_{E}\left(\boldsymbol{i}_{E}\right)\right\}\right] .
$$

Therefore

$$
\sum_{\boldsymbol{\alpha}^{(q)} \in \overline{\boldsymbol{C}}_{J^{(-q)}}} \cdots \sum_{\boldsymbol{\alpha}^{(p)} \in \overline{\boldsymbol{C}}_{J}^{(-p)}} \prod_{l \in J} \frac{\pi_{c_{k} i_{l} \alpha_{j}^{(l)}}^{\left(\bar{J}\{l l\}, J^{(-l)}\right)}}{\pi_{\boldsymbol{i}}}
$$

never has $\exp \left(\theta_{E^{*}}\left(\boldsymbol{i}_{E^{*}}^{*}\right)\right)$ as a factor of any terms of the summand, instead replacing these with $\exp \left(\theta_{E^{*}}\left(\tilde{\boldsymbol{i}}_{E^{*}}^{(l)}\right)\right.$ (which may be one), whereas

$$
\sum_{\boldsymbol{\alpha}_{q} \in \overline{\boldsymbol{C}}_{J}} \cdots \sum_{\boldsymbol{\alpha}_{p-1} \in \overline{\boldsymbol{C}}_{J}} \prod_{l \in J^{(-p)}} \frac{\pi_{c_{k} \alpha_{l j}}^{(\bar{J}, J)}}{\pi_{\boldsymbol{i}}}
$$

includes $\exp \left(m\left(\boldsymbol{\alpha}^{(q)}, \ldots, \boldsymbol{\alpha}^{(p)}\right) \theta_{E^{*}}\left(\boldsymbol{i}_{E^{*}}^{*}\right)\right)$ as a factor of every summand, with $m\left(\boldsymbol{\alpha}^{(q)}, \ldots, \boldsymbol{\alpha}^{(p)}\right)=\left|\left\{l: \alpha_{l q}=i_{q}^{*}\right\}\right|$. Therefore there exists $\boldsymbol{i} \in \tilde{\mathcal{I}}_{V}$ for which $\operatorname{Pr}(\boldsymbol{y}=\boldsymbol{i} \mid A) \neq \prod_{j} \operatorname{Pr}\left(y_{j}=i_{j} \mid A\right)$, completing the proof.

It follows that for any $H \in \mathscr{H}$ meeting the conditions of Remark 3.4, the only smaller partitions satisfying conditional independence that can be formed from elements of $\mathcal{P}_{H}^{0}$ are obtained by replacing events in the collections $\mathcal{P}_{H, A}^{\alpha}$ with events $\mathcal{B}^{\alpha}$. Therefore, noting that every partition of the sample space can be formed from unions of events in the partition consisting solely of Cartesian products of singletons, it follows that every partition satisfying conditional indepndence has at least the number of events given in (14), so long as the conditions of Remark 3.4 are satisfied.

1.2. Constructive nonnegative matrix rank result. The following proposition shows that in the two-dimensional case, the nonnegative rank can be bounded by one plus the minimum number of rows and columns that 
contain all of the cells that differ from a rank one nonnegative matrix. Figure S.1 shows several examples of the essential principle the proof, which is constructive. Although in the case of probability tensors corresponding to log-linear models, this result is a corollary of Theorem 3.2, the constructive approach is very instructive and provided intuition for the general result.

Proposition 1.1. Suppose $M$ is a $d \times d$ nonnegative matrix. Let $\lambda^{(1)}, \lambda^{(2)}$ be nonnegative vectors and set $\tilde{M}=\lambda^{(1)} \otimes \lambda^{(2)}$ with

$$
\begin{aligned}
& C_{M}=\left\{\left(c_{1}, c_{2}\right): M_{c_{1} c_{2}}-\tilde{M}_{c_{1} c_{2}} \neq 0\right\}, \quad C_{M}^{(1)}=\left\{c_{1}:\left(c_{1}, c_{2}\right) \in C_{M}\right\} \\
& C_{M}^{(2)}=\left\{c_{2}:\left(c_{1}, c_{2}\right) \in C_{M}\right\},
\end{aligned}
$$

and $\mathscr{H}=\left\{H: T_{\left(C_{M}, H\right)}=C_{M}\right\}$. Define $|H|=\left|H_{1}\right|+\left|H_{2}\right|$. Then $r n k_{P}^{+}(M) \leqslant$ $1+\bigwedge_{H \in \mathscr{H}}|H|$.

Proof. Let $H=\left(H_{1}, H_{2}\right)$ be any element of $\mathscr{H}$. Set

$$
\begin{aligned}
& \lambda_{0 c_{1}}^{(1)}=\lambda^{(1)} \mathbb{1}\left(c_{1} \notin H_{1}\right), \text { and } \\
& \lambda_{0 c_{2}}^{(2)}=\lambda^{(2)} \mathbb{1}\left(c_{2} \notin H_{2}\right),
\end{aligned}
$$

and put $M^{(0)}=\lambda_{0}^{(1)} \otimes \lambda_{0}^{(2)}$. Then for $1 \leqslant h \leqslant\left|H_{1}\right|$ set

$$
\begin{aligned}
& \lambda_{h c_{1}}^{(1)}=\mathbb{1}\left(c_{1}=H_{1 h}\right), \text { and } \\
& \lambda_{h c_{2}}^{(2)}=M_{H_{1 h} c_{2}},
\end{aligned}
$$

where $H_{1 h}$ is the $h$ th element of (any ordering of) $H_{1}$. Then set $M^{(1)}=$ $\sum_{h=1}^{\left|H_{1}\right|} \lambda_{h}^{(1)} \otimes \lambda_{h}^{(2)}$. Finally for $1 \leqslant h \leqslant\left|H_{2}\right|$ set

$$
\begin{aligned}
& \lambda_{h c_{1}}^{(1)}=M_{c_{1} H_{2 h}} \mathbb{1}\left(c_{1} \notin H_{1}\right) \text { and }, \\
& \lambda_{h c_{2}}^{(2)}=\mathbb{1}\left(c_{2}=H_{2 h}\right),
\end{aligned}
$$

and put $M^{(2)}=\sum_{h=1}^{\left|H_{1}\right|} \lambda_{h}^{(1)} \otimes \lambda_{h}^{(2)}$. Then $M^{(0)}+M^{(1)}+M^{(2)}=M$ and therefore $M$ has a $1+|H|=1+\bigwedge_{H^{\prime} \in \mathscr{H}}\left(\left|H^{\prime}\right|\right)$-term nonnegative PARAFAC expansion.

2. Posterior computation for c-Tucker models. The conditional posteriors for all the parameters can be derived in closed form using standard algebra and the sampler cycles through the following steps, 


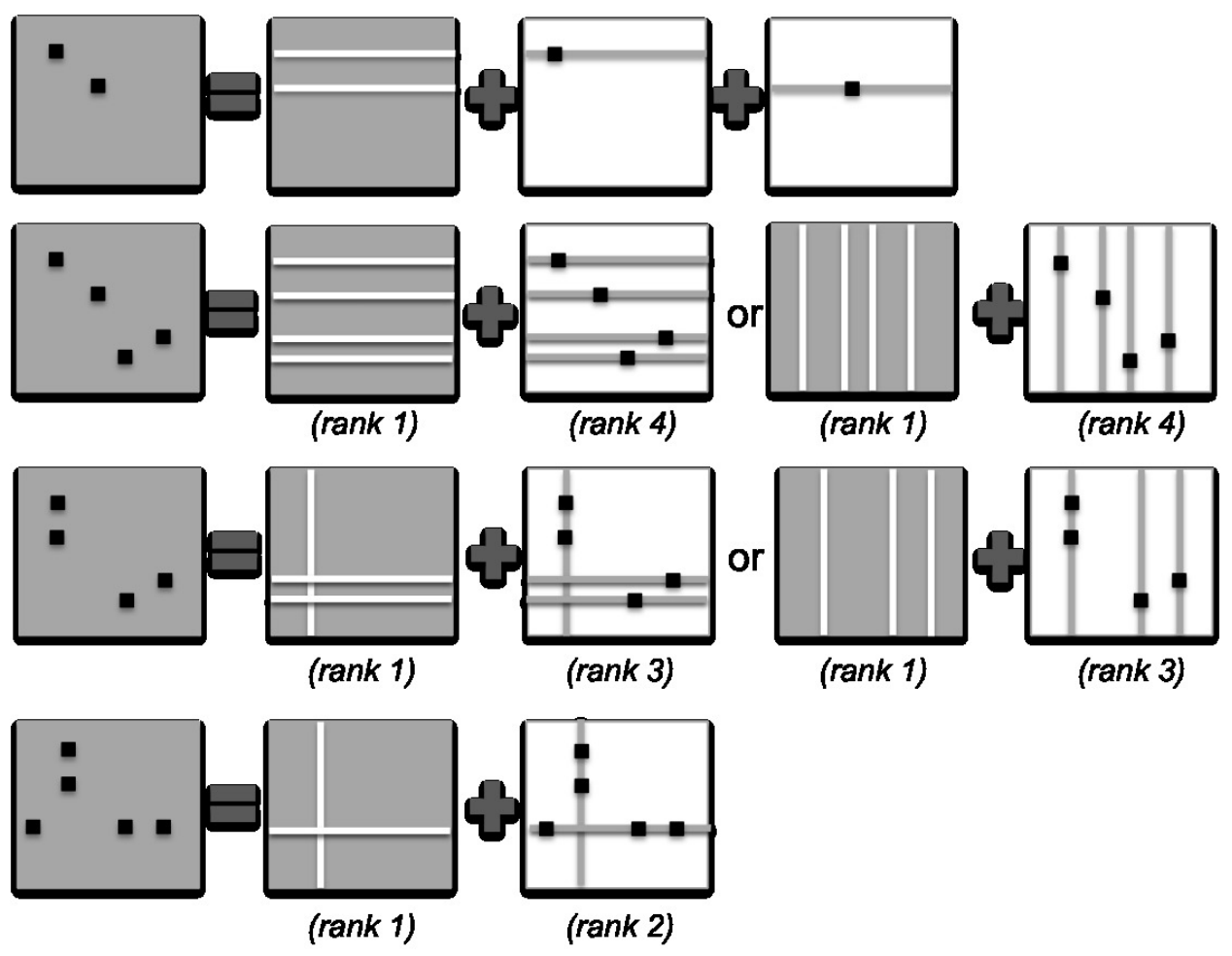

FIG S.1. Examples of nonnegative PARAFAC expansions for matrices. Black indicates cells containing interaction terms, gray indicates cells that do not contain interaction terms, and white indicates cells containing zeros.

Step 1. For $j: s_{j}=s$ and $h=1, \ldots, m$, update $\boldsymbol{\lambda}_{h}^{(j)}$ from the following Dirichlet full conditional posterior distribution,

$$
\pi\left(\boldsymbol{\lambda}_{h}^{(j)} \mid-\right) \sim \operatorname{Diri}\left(a_{j 1}+\sum_{i: z_{i s}=h} 1\left(y_{i j}=1\right), \ldots, a_{j d_{j}}+\sum_{i: z_{i s}=h} 1\left(y_{i j}=d_{j}\right)\right) .
$$

Step 2. Sample the latent class indicators $z_{i s}$ for $s \in\{1, \ldots, k\}$ from the following full conditional distribution,

$$
\operatorname{pr}\left(z_{i s}=h_{s} \mid-\right) \propto\left(\prod_{j: s_{j}=s} \lambda_{h_{s} y_{i j}}^{(j)}\right) \psi_{w_{i} h_{s}}^{(s)}, h_{s}=1, \ldots, m .
$$

Step 3. Sample $w_{i}$ from the following full-conditional distribution,

$$
\operatorname{pr}\left(w_{i}=l \mid-\right) \propto \nu_{l} \prod_{s=1}^{k} \psi_{l z_{i s}}^{(s)}, l=1, \ldots, k .
$$


Step 4. Sample $\nu_{l}^{*}$ from the following full-conditional distribution,

$$
\pi\left(\nu_{l}^{*} \mid-\right) \sim \operatorname{beta}\left(1+m_{l}, \beta+m_{l+}\right) l=1, \ldots, k,
$$

where $m_{l}=\sum_{i=1}^{n} 1\left(w_{i}=l\right)$ and $m_{l+}=\sum_{i=1}^{n} 1\left(w_{i}>l\right)$.

Step 5. To update $\phi_{l h}^{(s)}$ for $s \in\{1, \ldots, k\}$ define $n_{l h}^{(s)}=\sum_{i: w_{i}=l} 1\left(z_{i s}=h\right)$ and $n_{l h+}^{(s)}=\sum_{i: w_{i}=l} 1\left(z_{i s}>h\right)$. Then, the full conditional posterior of $\phi_{l h}^{(s)}$ is

$$
\pi\left(\phi_{l h}^{(s)} \mid-\right) \sim \operatorname{Beta}\left(1+n_{l h}^{(s)}, \delta_{1}+n_{l h+}^{(s)}\right)
$$

Step 6. Assuming a gamma $\left(a_{\beta}, b_{\beta}\right)$ prior for $\beta$, the full conditional posterior is

$$
\pi(\beta \mid-) \sim \operatorname{gamma}\left(a_{\beta}+k, b_{\beta}-\sum_{l=1}^{k} \log \left(1-\nu_{l}^{*}\right)\right) .
$$

Step 7. Assuming a gamma $\left(a_{\delta}^{(s)}, b_{\delta}^{(s)}\right)$ prior for $\delta_{s}$ for each $s \in\{1, \ldots, k\}$, the full conditional posterior is

$$
\pi\left(\delta_{1} \mid-\right) \sim \operatorname{gamma}\left(a_{\delta}^{(s)}+m k, b_{\delta}^{(s)}-\sum_{l=1}^{k} \sum_{h=1}^{m} \log \left(1-\phi_{l h}^{(s)}\right)\right) .
$$

Step 8. The groups $s_{j}$ are updated sequentially. Set $L_{i j l}=\operatorname{Pr}\left(y_{j}=y_{i j} \mid s_{j}=\right.$ $l)=\lambda_{z_{l} y_{j}}^{(j)}$, and $L_{j l}=\prod_{i} L_{i j l}$. Then sample $s_{j}$ as

$$
\pi\left(s_{j}=l \mid-\right)=\frac{\xi_{l} L_{j l}}{\sum_{l=1}^{k} \xi_{l} L_{j l}} .
$$

Step 9 . Let $n_{l}=\sum_{j} \mathbb{1}_{s_{j}=l}$, and sample $\xi$ from

$$
p(\xi \mid-) \sim \operatorname{Dirichlet}\left(n_{1}+1 / k, \ldots, n_{k}+1 / k\right) \text {. }
$$

\section{Supplemental figures and tables for section 5 .}




\begin{tabular}{|c|c|c|c|c|c|c|c|c|c|c|c|c|c|c|c|c|}
\hline & \multicolumn{6}{|c|}{ ADL } & \multicolumn{10}{|c|}{ IADL } \\
\hline & 1 & 2 & 3 & 4 & 5 & 6 & 1 & 2 & 3 & 4 & 5 & 6 & 7 & 8 & 9 & 10 \\
\hline \multicolumn{17}{|l|}{ ADL } \\
\hline 1 & - & 1.00 & 1.00 & 0.00 & 0.00 & 0.37 & 1.00 & 1.00 & 1.00 & 0.00 & 0.00 & 0.00 & 1.00 & 0.00 & 0.00 & 1.00 \\
\hline 2 & 0.21 & - & 1.00 & 0.00 & 0.00 & 1.00 & 1.00 & 1.00 & 1.00 & 0.00 & 0.99 & 0.99 & 1.00 & 0.01 & 1.00 & 1.00 \\
\hline 3 & 0.26 & 0.25 & - & 1.00 & 0.00 & 1.00 & 1.00 & 1.00 & 1.00 & 0.00 & 0.25 & 0.17 & 1.00 & 0.00 & 0.68 & 1.00 \\
\hline 4 & 0.06 & 0.09 & 0.12 & - & 1.00 & 1.00 & 1.00 & 1.00 & 1.00 & 1.00 & 0.05 & 1.00 & 0.06 & 1.00 & 0.99 & 0.00 \\
\hline 5 & 0.03 & 0.06 & 0.05 & 0.20 & - & 1.00 & 0.99 & 0.99 & 0.97 & 1.00 & 0.98 & 1.00 & 0.36 & 1.00 & 1.00 & 0.00 \\
\hline 6 & 0.10 & 0.14 & 0.18 & 0.38 & 0.21 & - & 1.00 & 1.00 & 1.00 & 1.00 & 0.99 & 1.00 & 1.00 & 1.00 & 1.00 & 0.00 \\
\hline \multicolumn{17}{|c|}{ IADL } \\
\hline 1 & 0.20 & 0.27 & 0.25 & 0.16 & 0.11 & 0.27 & - & 1.00 & 1.00 & 1.00 & 1.00 & 1.00 & 1.00 & 1.00 & 1.00 & 1.00 \\
\hline 2 & 0.14 & 0.20 & 0.19 & 0.20 & 0.15 & 0.32 & 0.42 & - & 1.00 & 1.00 & 0.99 & 1.00 & 1.00 & 1.00 & 1.00 & 1.00 \\
\hline 3 & 0.18 & 0.24 & 0.20 & 0.13 & 0.11 & 0.22 & 0.48 & 0.37 & - & 1.00 & 1.00 & 1.00 & 1.00 & 1.00 & 1.00 & 1.00 \\
\hline 4 & 0.04 & 0.08 & 0.08 & 0.19 & 0.19 & 0.25 & 0.14 & 0.21 & 0.12 & - & 0.04 & 1.00 & 0.00 & 1.00 & 0.99 & 0.00 \\
\hline 5 & 0.08 & 0.14 & 0.10 & 0.09 & 0.13 & 0.14 & 0.20 & 0.17 & 0.22 & 0.09 & - & 1.00 & 1.00 & 1.00 & 1.00 & 0.99 \\
\hline 6 & 0.07 & 0.12 & 0.10 & 0.13 & 0.18 & 0.19 & 0.19 & 0.21 & 0.19 & 0.15 & 0.22 & - & 1.00 & 1.00 & 1.00 & 1.00 \\
\hline 7 & 0.15 & 0.22 & 0.15 & 0.09 & 0.10 & 0.15 & 0.30 & 0.22 & 0.32 & 0.09 & 0.31 & 0.21 & - & 1.00 & 1.00 & 1.00 \\
\hline 8 & 0.05 & 0.09 & 0.07 & 0.13 & 0.37 & 0.17 & 0.16 & 0.18 & 0.18 & 0.14 & 0.26 & 0.25 & 0.21 & - & 1.00 & 0.99 \\
\hline 9 & 0.09 & 0.14 & 0.10 & 0.11 & 0.19 & 0.16 & 0.22 & 0.21 & 0.24 & 0.11 & 0.30 & 0.24 & 0.31 & 0.41 & - & 1.00 \\
\hline 10 & 0.17 & 0.19 & 0.15 & 0.06 & 0.05 & 0.09 & 0.22 & 0.15 & 0.24 & 0.05 & 0.21 & 0.13 & 0.33 & 0.12 & 0.21 & - \\
\hline
\end{tabular}

Estimated Cramér's $V$ associations (elements under the main diagonal) and posterior probabilities $\operatorname{Pr}\left(H_{1, \rho} \mid y^{(1: n)}\right)$ (elements above the main diagonal) in the NLTCS data estimated using the c-Tucker model. 


\begin{tabular}{|c|c|c|c|c|c|c|c|c|c|c|c|c|c|c|c|c|}
\hline & \multicolumn{6}{|c|}{ ADL } & \multicolumn{10}{|c|}{ IADL } \\
\hline & 1 & 2 & 3 & 4 & 5 & 6 & 1 & 2 & 3 & 4 & 5 & 6 & 7 & 8 & 9 & 10 \\
\hline \multicolumn{17}{|l|}{ ADL } \\
\hline 1 & - & 1.00 & 1.00 & 0.00 & 0.00 & 0.61 & 1.00 & 1.00 & 1.00 & 0.00 & 0.00 & 0.00 & 0.99 & 0.00 & 0.00 & 1.00 \\
\hline 2 & 0.21 & - & 1.00 & 0.43 & 0.00 & 1.00 & 1.00 & 1.00 & 1.00 & 0.00 & 0.99 & 1.00 & 1.00 & 0.08 & 1.00 & 1.00 \\
\hline 3 & 0.26 & 0.25 & - & 1.00 & 0.00 & 1.00 & 1.00 & 1.00 & 1.00 & 0.00 & 0.05 & 0.45 & 1.00 & 0.00 & 0.14 & 0.99 \\
\hline 4 & 0.07 & 0.10 & 0.15 & - & 1.00 & 1.00 & 1.00 & 1.00 & 1.00 & 1.00 & 0.38 & 1.00 & 0.32 & 1.00 & 0.98 & 0.00 \\
\hline 5 & 0.03 & 0.06 & 0.05 & 0.21 & - & 1.00 & 0.99 & 1.00 & 0.98 & 1.00 & 1.00 & 1.00 & 0.32 & 1.00 & 1.00 & 0.00 \\
\hline 6 & 0.10 & 0.14 & 0.20 & 0.38 & 0.21 & - & 1.00 & 1.00 & 1.00 & 1.00 & 1.00 & 1.00 & 1.00 & 1.00 & 1.00 & 0.03 \\
\hline \multicolumn{17}{|c|}{ IADL } \\
\hline 1 & 0.21 & 0.28 & 0.28 & 0.18 & 0.11 & 0.28 & - & 1.00 & 1.00 & 1.00 & 1.00 & 1.00 & 1.00 & 1.00 & 1.00 & 1.00 \\
\hline 2 & 0.14 & 0.19 & 0.19 & 0.21 & 0.16 & 0.34 & 0.43 & - & 1.00 & 1.00 & 1.00 & 1.00 & 1.00 & 1.00 & 1.00 & 1.00 \\
\hline 3 & 0.16 & 0.22 & 0.18 & 0.13 & 0.11 & 0.22 & 0.48 & 0.40 & - & 1.00 & 1.00 & 1.00 & 1.00 & 1.00 & 1.00 & 1.00 \\
\hline 4 & 0.04 & 0.08 & 0.08 & 0.19 & 0.18 & 0.25 & 0.15 & 0.23 & 0.13 & - & 0.33 & 1.00 & 0.13 & 1.00 & 1.00 & 0.00 \\
\hline 5 & 0.06 & 0.12 & 0.09 & 0.10 & 0.14 & 0.14 & 0.18 & 0.17 & 0.19 & 0.10 & - & 1.00 & 1.00 & 1.00 & 1.00 & 1.00 \\
\hline 6 & 0.06 & 0.12 & 0.10 & 0.14 & 0.19 & 0.20 & 0.19 & 0.21 & 0.18 & 0.17 & 0.27 & - & 1.00 & 1.00 & 1.00 & 1.00 \\
\hline 7 & 0.13 & 0.21 & 0.14 & 0.10 & 0.10 & 0.14 & 0.27 & 0.21 & 0.28 & 0.09 & 0.30 & 0.23 & - & 1.00 & 1.00 & 1.00 \\
\hline 8 & 0.05 & 0.09 & 0.07 & 0.14 & 0.39 & 0.19 & 0.16 & 0.19 & 0.17 & 0.15 & 0.26 & 0.26 & 0.20 & - & 1.00 & 0.95 \\
\hline 9 & 0.07 & 0.13 & 0.09 & 0.11 & 0.20 & 0.16 & 0.20 & 0.21 & 0.23 & 0.12 & 0.31 & 0.25 & 0.30 & 0.42 & - & 1.00 \\
\hline 10 & 0.14 & 0.16 & 0.13 & 0.06 & 0.05 & 0.09 & 0.20 & 0.13 & 0.20 & 0.05 & 0.19 & 0.12 & 0.32 & 0.11 & 0.20 & - \\
\hline
\end{tabular}

Estimated Cramér's $V$ associations (elements under the main diagonal) and posterior probabilities $\operatorname{Pr}\left(H_{1, \rho} \mid y^{(1: n)}\right)$ (elements above the main diagonal) in the NLTCS data estimated using copula Gaussian graphical model in [1]. 


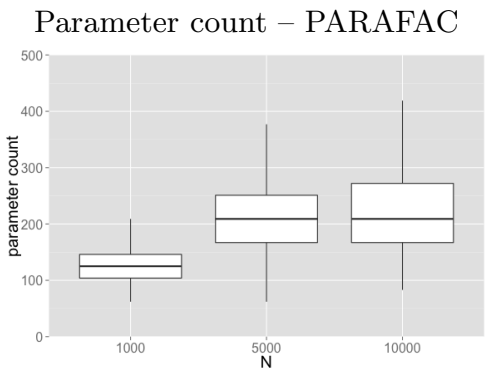

Parameter count - c-Tucker

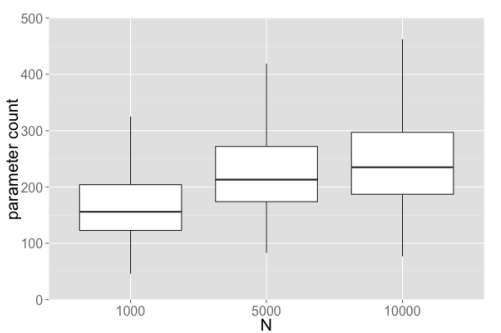

FIG S.2. Left figure: box plot of parameter count for PARAFAC model for the simulation study based on example 4.3. Right figure: box plot of parameter count for c-Tucker model for the same simulation study.

\section{References.}

[1] Adrian Dobra and Alex Lenkoski. Copula gaussian graphical models and their application to modeling functional disability data. The Annals of Applied Statistics, 5(2A): 969-993, 2011.

114 Old Chemistry Building

DUKE UNIVERSITY

Durham, NC 27708

E-MAIL: james.johndrow@duke.edu

E-MAIL: dunson@duke.edu

URL: http://www.isds.duke.edu/ dunson/
155 Ireland Street

College Station, TX 77843

E-MAIL: anirbanb@stat.tamu.edu 